\title{
Effect of natural dye from beetroot juice on antioxidant properties and nutritional values of fondants
}

\author{
Amelia Buculei, Paulina Turcan
}

University Stefan cel Mare of Suceava, Suceava, Romania

Keywords:

Fondant

Beetroot

Dye

Antioxidant

Betaine

Article history:

Received 20.09.2017

Received in revised

form 22.11.2017

Accepted 29.12.2017

Corresponding author:

Paulina Turcan

E-mail:

paulina.turcan@

outlook.com

\section{Abstract}

Introduction. It was performed a research the physical and chemical, antioxidant and nutritional properties of the fondants with beetroot juice addition.

Materials and methods. 3 samples of fondants with juice obtained from raw (1), boiled (2) and baked (3) beetroot were used in this study. Methods: sensory analysis with the help of 15 subjects, the antioxidant activity assessment through DPPH method, and the chemical test, which refers to moisture, ash, mineral and reducing sugar determinations.

Results and discussions. Sample 1, which are fondant candies with juice obtained from fresh beetroot, was the most appreciated with a total ranking of 8,92, and sample III, fondant candies with juice obtained from baked beetroot, was the least appreciated with a result of 8,55. Sample II, fondant candies with juice obtained from boiled beetroot, had an overall assessment of 8,59.

Highest antioxidant capacity occurs in the case of fondant candies with red juice obtained from raw beetroots (sample 1), with an overall performance of 93,22\%. A rather lower result had the sample 2 with $92,84 \%$. Sample 3 showed the lowest result of $91,49 \%$.

The highest results in the matter of moisture content $(94,20 \%)$ and reducing sugars $(14,20 \%)$ had the sample 3. On the contrary, the lowest results for both moisture content and reducing sugars was shown by sample 2 with $93,2 \%$ and $12,1 \%$.

The highest value of ash content was shown by the sample with raw beetroot juice, 5,66\%. Sample 3 had the lowest level of ash content, $1,18 \%$, five times lower comparing to the first sample.

Conclusions. The fondants obtained from raw beetroot juice has the most pronounced antioxidant capacity and the betaine content is almost double compared with other analysed samples.

DOI: $10.24263 / 2310-$

1008-2017-5-2-6 


\section{Introduction}

Fondants are types of confectionery. They are categorized based products called fondanterie flux. Besides fondants in this category are included sherbet, marzipan and other confectionery [6].

Natural dye is used in both commercial production of food and the cooking class. Due to their safety and general availability, natural dyes are used in a variety of non-food applications, for example in handicrafts and educational kits. By the mid 1800s the only source of dyes used in foods were natural, extracted from saffron, carrots, dude, beetroot etc. [2] [3].

Manufacturers continue to seek solutions for natural ingredients for the development of new products, particularly for products that are marketed as "better for consumption". As costumers demand for healthy food and healthy increases, so does the use of natural colors [1] [4].

Also, natural colors must be declared on the product label. If the red beet juice is used to shade a foodstuff (sweets, jellies, etc.), it should be indicated as an additive color [4] [5].

It was discovered that in 2010 only $1.6 \%$ of confectionery products contained natural colorants; this number has increased and is estimated to account for over $10 \%$ of all confections in 2020 [13].

An interesting use of natural coloring aids is that many are bioactive and have a strong antioxidant capacity; for example, betaine extracted from red beet is one of the most powerful natural antioxidants [2].

A study by a group of UK researchers in 2012 compared the antioxidant activity of betaine extracted from red beetroot Detroit Dark Red, with the antioxidant capacity of betaine extracted from an unknown variety.

It has been found that betaine extract obtained from the Detroit Dark Red variety has higher salt antioxidant capacity (DPPH) than the other extract; 3.28 times greater packing capacity, and ORACs 20 times higher.

It has been demonstrated in the same study that betaine remains stable in the gastrointestinal tract without any significant loss of antioxidant properties, which makes its value as a food additive increase.

Betaines are divided into two groups: betaxanthines and beta-cyanines, due to their chemical structure. Betaxanthines are condensation products of betalamic acid, they have a deep violet color [12].

The purpose of this research is the assessment of the physical and chemical behavior of the beetroot juice added in the product, as well as its properties changes after heat treatment application. Plus, another intent of this paper is to find if there can be found any correlation between sensorial and chemical performances of the samples.

\section{Materials and methods}

For this study were analysed the following samples:

Sample 1 - fondant candies with beetroot juice, obtained from fresh beetroot;

Sample 2 - fondant candies with beetroot juice, obtained from boiled beetroot;

Sample 3 - fondant candies with beetroot juice, obtained from baked beetroot. 


\section{Sensory analysis}

For the sensory analysis has been used the method with hedonic scale (hedonic test). This method is used for the purpose of introducing a new product on the market, by assessing the consumer's reaction to the organoleptic properties of the product [7].

The hedonic test allows to determinate both the best sample and the degree of preference of certain factors such as the packing method, the modification of the recipe as well as the shelf life [7].

For this method 15 subjects were selected with the help of which were performed the following determinations: appearance, colour, taste, smell, flavour and texture. [7]

The experiment was held in the laboratory with specific conditions of light, ventilation and temperature.

\section{Antioxidant activity}

This test was performed using the DPPH reagent and a spectrophotometer.

DPPH (2,2-diphenyl-1-picrylhydrazyl) is one of the most stable and commercially available organic radionuclides and has a maximum UV-VIS absorption at $517 \mathrm{~nm}$. DPPH method is based on the generation of free radicals, from a methanol solution of 2,2diphenyl -1- picrylhydrazyl, which absorption disappears in the presence of an antioxidant [8]

Preparation of the solid samples. In three volumetric flasks were introduced $2,5 \mathrm{mg}$ of each sample, followed by bringing to the mark with methanol. The flasks were agitated vigorously and left in the dark for 15 minutes, after which the content was filtered through filter paper. The obtained extracts were used for the calibration curve [8].

Establishment of the calibration curve. For this determination, firstly, was used a blank sample, namely a methanol solution, used as a standard, for which was determined the absorbance $\left(\mathrm{A}_{\text {standard }}\right)$. The calibration curve for each sample was traced based on 10 solutions containing different amounts of the certain sample extracts and methanol, to which was added $500 \mu \mathrm{l}$ of DPPH reagent in every test tube right before the cuvette should have been placed in the spectrophotometer. These manipulations were followed by the absorbance determination for each concentration $\left(\mathrm{A}_{\text {sample }}\right)[9]$.

Antioxidant activity value calculation. The inhibition percentage of the free radicals (I\%) was obtained with the equation [8]:

$$
I \%=\frac{A_{s \tan d a r t}-A_{\text {sample }}}{A_{s \tan \text { dart }}} 100
$$

\section{Chemical analysis}

Moisture determination. Moisture content of each fondant candy with beets juice was performed by using the digital moisture analyzer at $100^{\circ} \mathrm{C}$. The total soluble solids were determined using a Digital ABBE Refractometer and expressed using a Brix degree scale $\left({ }^{\circ} \mathrm{B}\right)[10]$.

Ash determination. The classic calcination method was used. In a porcelain crucible, weighed in advance, was introduced the sample. The crucible was placed on the flame of a gas lamp until the smoke appeared [11].

This procedure was followed by the insertion of the crucible in the electric furnace set 
at $750^{\circ} \mathrm{C}$ and left the necessary time there until a pale white or gray residue with no traces of charcoal was obtained [11].

The crucible was cooled in a desiccator until it reached the room temperature, then it was weighed.

The ash content was determined with the formula [11]:

$$
A s h=\frac{m_{1}}{m} 100
$$

where $\mathrm{m}_{1}$ - is the amount of ash in $\mathrm{g}$, which is deduced from the difference between the weight of the crucible with ash after calcination and the empty crucible

$\mathrm{m}$ - the amount of sample taken into work, this being calculated from the difference between the weight of the crucible with sample before calcination and the empty crucible [11]

Quantitative determination of mineral substances (EDX spectroscope). Rontgen energy-dispersive fluorescence spectroscopy is based on measuring discrete energy of each Rontgen wavelength as expression of concentration and composition. With the emergence of discrete radiant energy on a semiconductor detector it produces a certain number of pairs of voids and through them a certain electrical pulse. Current pulses, according to the current value, are distributed by a multichannel analyzer (about 1000 channels) and give a specific spectrum that has the order of pulses and the energy abscissa.

All Rontgen fine analysis procedures, whether refractive or spectroscopic, are analytical and control procedures that act completely non-destructively on the subject matter under investigation.

Determination of reducing sugar (Schoorl method). Reducing sugars reduce the copper-tartaric alkaline solution (Fehling's reagent) to $\mathrm{Cu}_{2} \mathrm{O}$, which is indirectly quantitatively determined by iodometric measurement of copper sulphate in the Fehling solution before and after reduction. The difference obtained is the amount of copper reduced by sugar.

- Extraction. An amount of 5-25g of the sample evenly blended, it is placed in a 150$200 \mathrm{ml}$ vial where was added $70-80 \mathrm{ml}$ of distilled water heated to $85-90{ }^{\circ} \mathrm{C}$, then placed on a water bath, and from the moment the water begins to boil for 30 minutes, started the stirring. The extract is filtered in a $100 \mathrm{ml}$ volumetric flask and washed with hot distilled water.

- Dosage of reducing sugars. In a $300 \mathrm{ml}$ Erlenmayer flask, $20 \mathrm{ml}$ of the flaky extract was introduced, then Fehling I solution $10 \mathrm{ml}$ and Fehling II solution $10 \mathrm{ml}$, the pot was heated on an asbestos sieve, the boiling time was exactly 2 minutes. The flask was cooled in a stream of water, then $20 \mathrm{ml}$ of potassium iodide solution and $15 \mathrm{ml}$ of sulfuric acid was added.

The liberated iodine is titrated with $0.1 \mathrm{~N}$ sodium thiosulfate in the presence of starch as an indicator. The solution of starch is added to the end of the titration when the solution had a pale yellow color. Titration is continued until the blue color disappears due to the presence of iodine. 
The amount of copper reduced by sugar is determined by the amount of $0.1 \mathrm{~N}$ sodium thiosulphate used for titration based on the equation:

$$
V=V_{1}-V_{2}
$$

where $\mathrm{V}_{1}$ - the volume of $0.1 \mathrm{~N}$ sodium thiosulphate used to titrate the blank sample (ml)

$\mathrm{V}_{2}$ - the volume of $0.1 \mathrm{~N}$ sodium thiosulphate used to titrate the actual sample $(\mathrm{ml})$

There is the amount of reducing sugar in the analyzed samples based on the equation:

$$
g \%=\frac{z \cdot d}{g} 100
$$

where $z$ is the quantity of inverted sugar from the corresponding table for $\mathrm{V} \mathrm{ml}$ of $\mathrm{Na}_{2} \mathrm{~S}_{2} \mathrm{O}_{3}$ $0,1 \mathrm{~N}$;

$d$ is dilution cipher;

$g$ is weight of analyzed sample (g).

\section{Results and discussions}

1. Sensory analysis

Following the sensory analysis on each of the three samples were obtained the following results.

Graphical interpretation of sensory analysis:

\subsection{Appearance assessment}

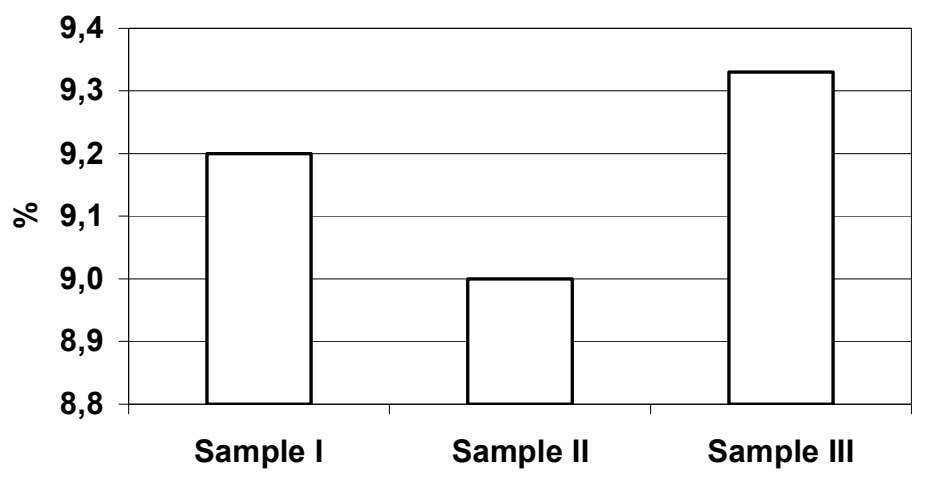

Figure 1.1. Appearance assessment of the fondant candies with beetroot juice

As results from this graph, sample III, namely candies containing juice obtained from baked beetroots, has the highest value for appearance 9,33. The lowest value of 9 has the second sample. 


\subsection{Colour assessment}

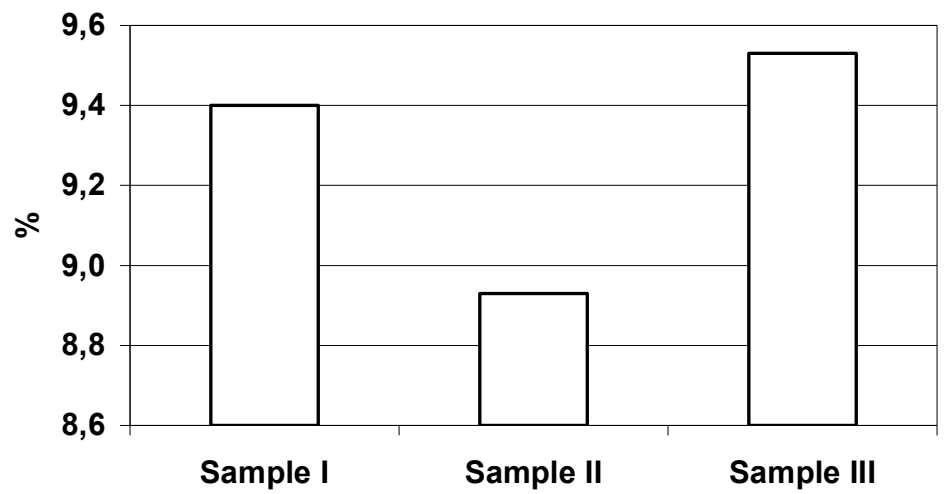

Figure 1.2. Colour assessment of the fondant candies with beetroot juice

Following the completion of the colour graph, sample III has the highest result of 9,53 compared to the other 2 samples.

\subsection{Taste assessment}

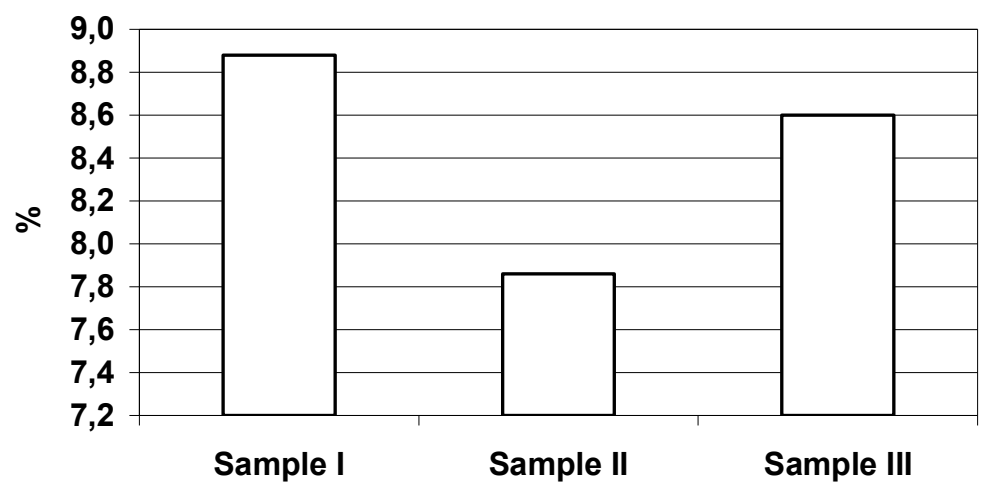

Figure 1.3. Taste assessment of the fondant candies with beetroot juice

Fondant candies with juice obtained from fresh beetroot have the highest grade in the taste category, 8,88. Meanwhile sample II was ranked with 7,86. 


\subsection{Smell assessment}

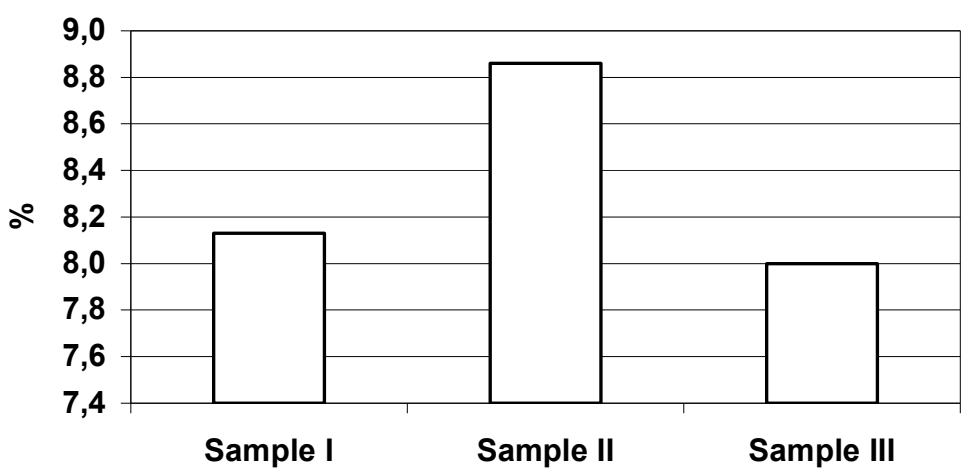

Figure1.4. Smell assessment of the fondant candies with beetroot juice

According to this graph, candies made with juice obtained from boiled beetroots are the most appreciated in terms of smell, with a 8,86 appreciation. Sample I and II had slightly the same results: 8,13 and 8 .

\subsection{Flavour assessment}

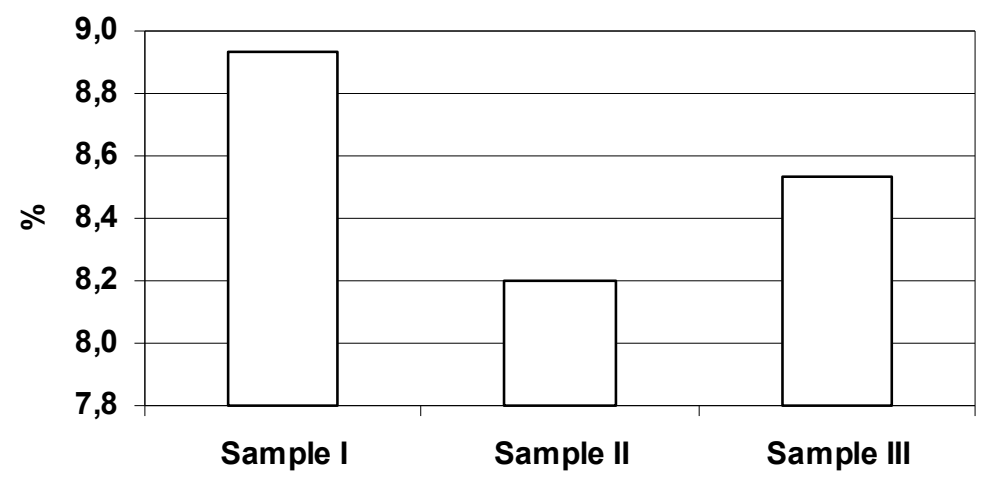

Figure1.5. Flavour assessment of the fondant candies with beetroot juice

According to graph 5, candies with juice obtained from raw beetroots have the most pronounced flavour, with a rank of 8,93. The lowest result, 8,20, was obtained by sample II. 


\subsection{Texture assessment}

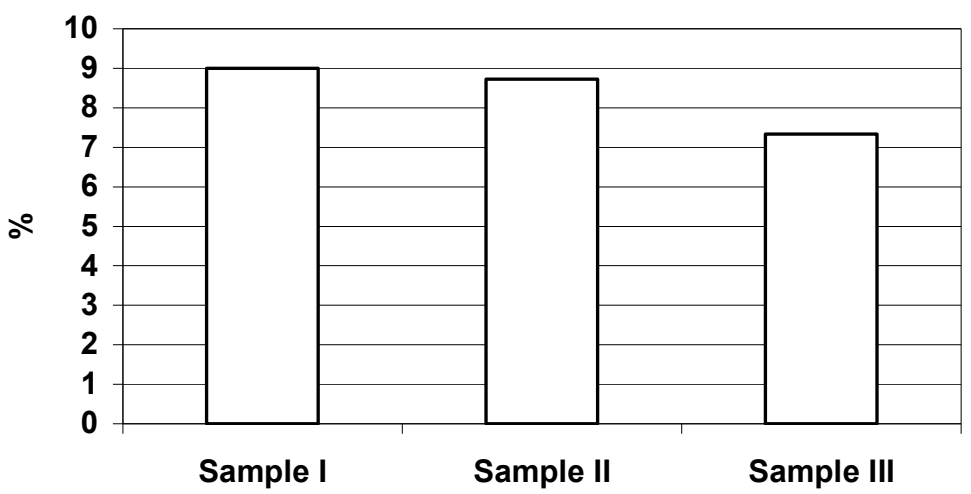

Figure 1.6. Texture assessment of the fondant candies with beetroot juice

In the matter of texture, samples were ranked as follows: 9 for sample I, 8,73 for sample II and 7,33 for sample III.

\subsection{Overall sensory analysis performance}

After performing the sensory analysis on all three different samples the following general graph was established:

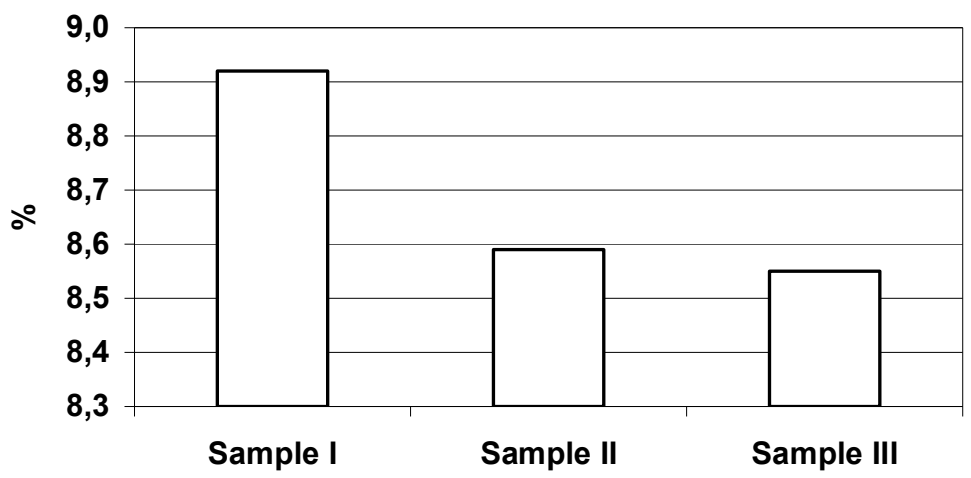

Figure 1.7. General graph of the sensory analysis performance

As a result of the overall chart, sample I was the most appreciated with a total ranking of 8,92 , and sample III was the least appreciated with a result of 8,55 . Sample II had an overall assessment of 8,59 . 
2. Antioxidant capacity determination

\subsection{Graphical interpretation of antioxidant capacity}

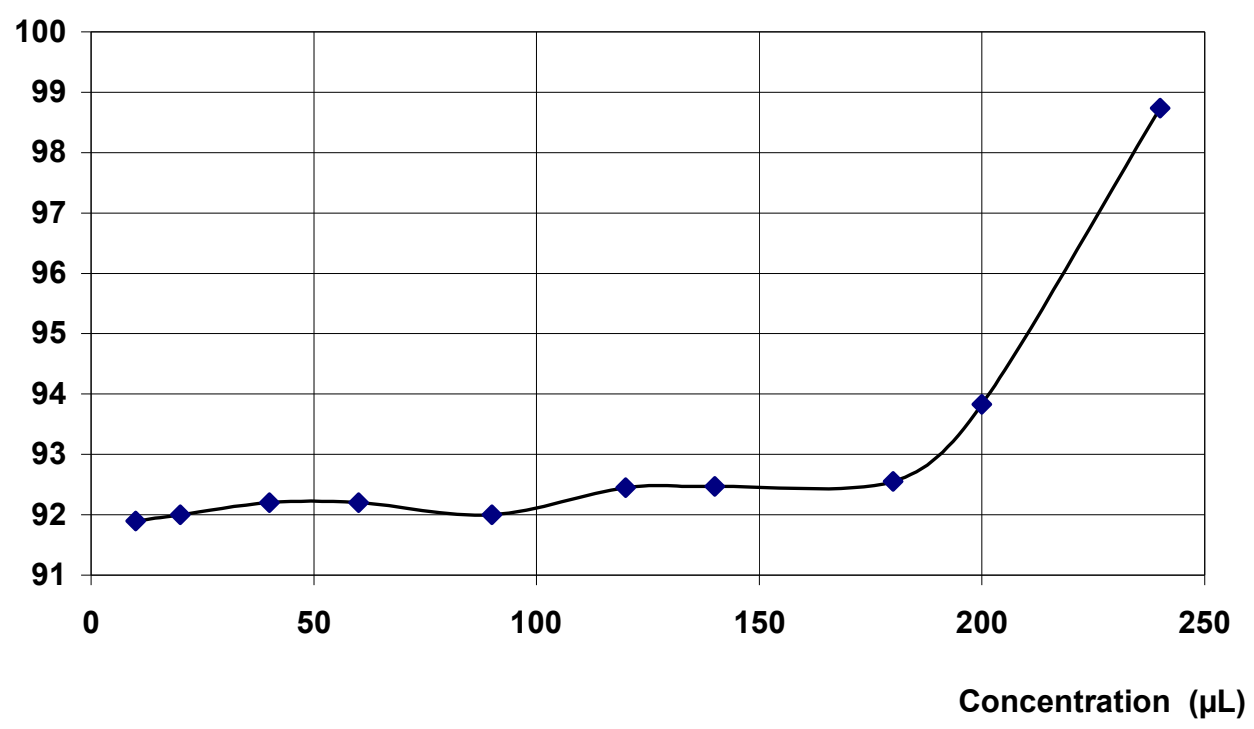

Figure 2.1.1. Sample 1 - Fondant candies with juice obtained from raw beetroots

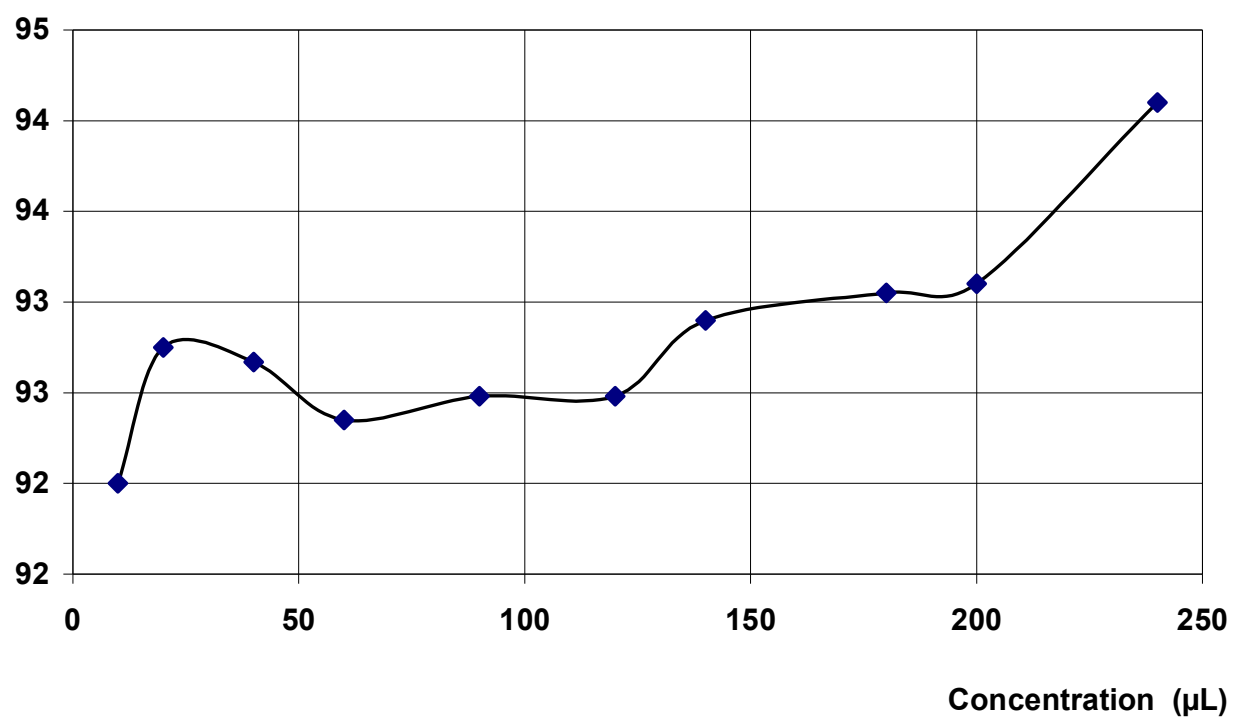

Figure 2.1.2. Sample 2 - Fondant candies with juice obtained from boiled beetroots 


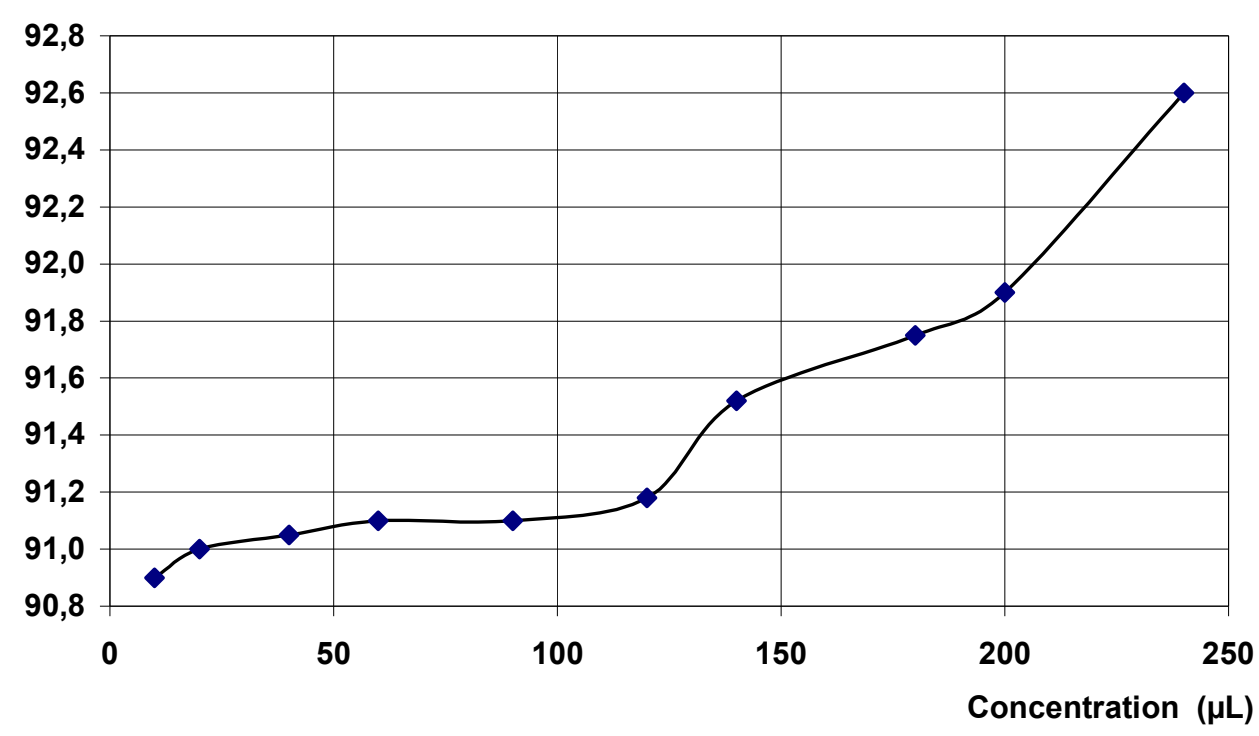

Figure 2.1.3. Sample 3 - Fondant candies with juice obtained from baked beetroots

\subsection{Overall antioxidant capacity performance}

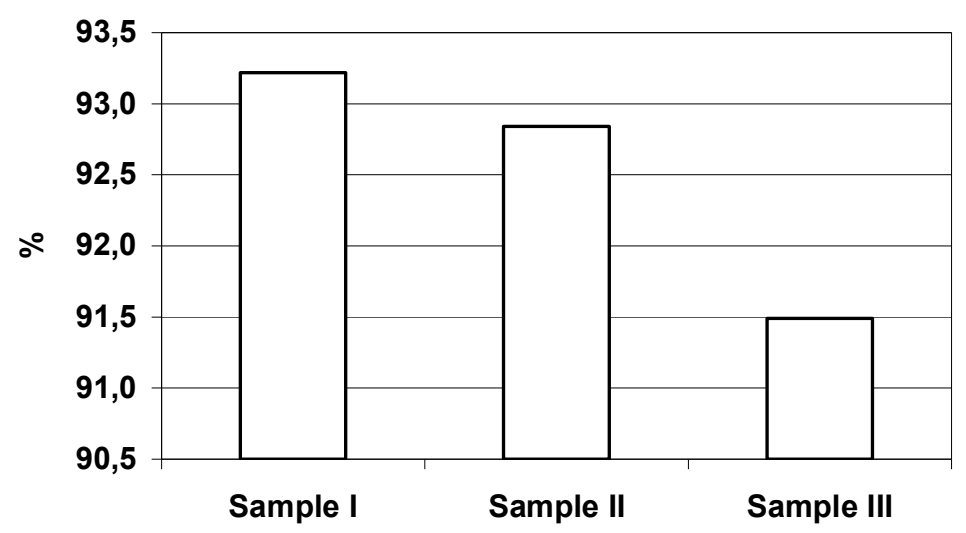

Figure 2.2.1. General graph of antioxidant capacity

The highest antioxidant capacity occurs in the case of fondant candies with red juice obtained from raw beetroots (sample I), with an overall performance of 93,22\%. A rather lower result had the sample II with $92,84 \%$. Simultaneously, sample III showed the lowest result of $91,49 \%$.

It is also noted that with the absorbance decrease the antioxidant activity decreases. 
3. Chemical analysis of the fondant candies with beetroot juice

\subsection{Moisture determination}

After keeping the samples in an oven at constant $100{ }^{\circ} \mathrm{C}$ for 5 hours, the following results were obtained:

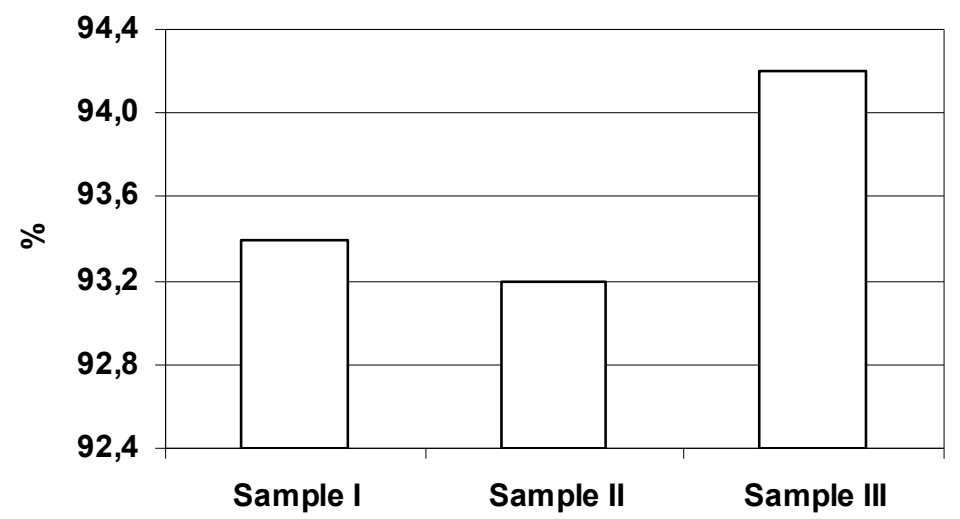

Figure 3.1. Graphical interpretation of moisture

Sample III has the highest result of $94,20 \%$, meanwhile samples I and II showed a slightly equal result of $93,4 \%$ and $93,2 \%$.

\subsection{Ash content}

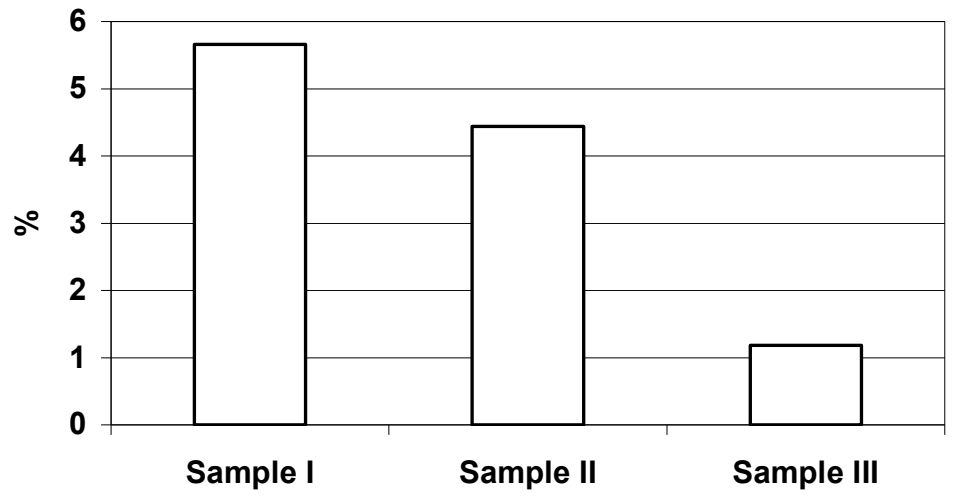

Figure 3.2. Graphical interpretation of ash content

As a result of this graph, sample I, fondant candies with juice obtained from raw beetroots has the highest ash content of 5,66\%. Sample III showed a five times lower result, $1,18 \%$. 


\subsection{Quantitative determination of mineral substances with EDX method}

Based on the quantitative determination of mineral substances from fresh beetroot juice were highlighted the following elements:

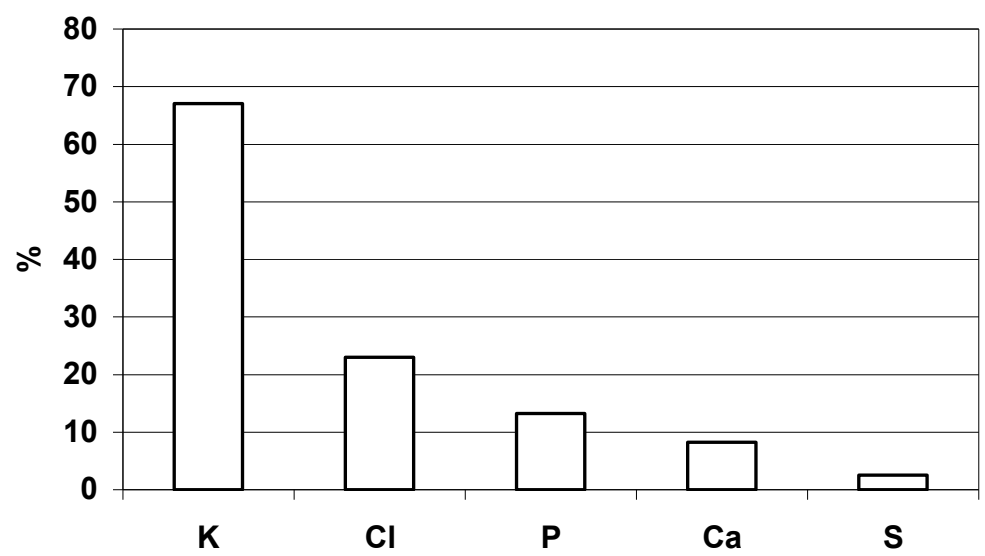

Figure 3.3.1. Graphical interpretation of mineral substances from fresh beetroot juice

According to the graph, following the quantitative determination of the mineral substances in the fresh beetroot juice, it contains an amount of $67,0 \% \mathrm{~K}$ that passes into ash, but also other elements such as: $\mathrm{Cl}(23.01 \%), \mathrm{P}(13,24 \%), \mathrm{Ca}(8.26 \%), \mathrm{S}(2.52 \%)$.

A significant proportion of these elements and minerals are found in the 3 fondant candy samples. The results are as follows:

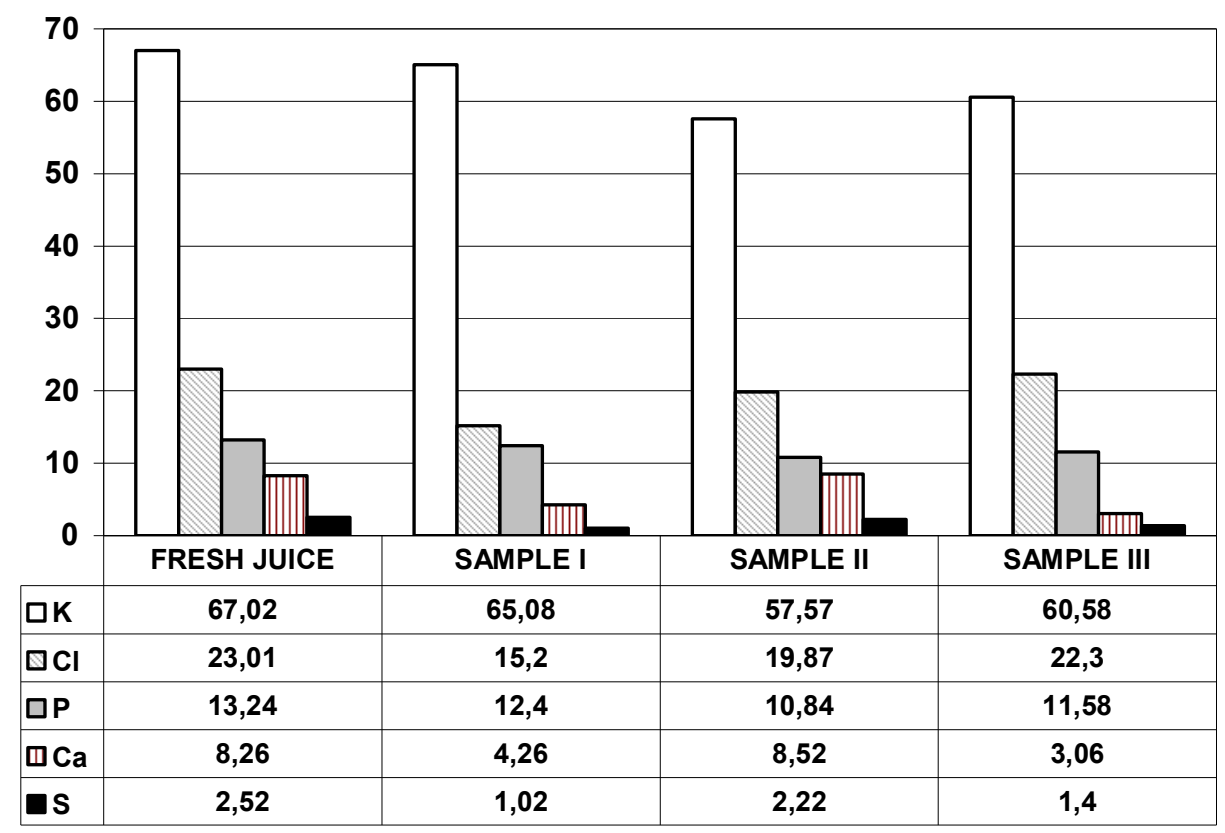

Figure 3.3.2. General graphic of mineral substances content 
Mineral substances pass into the three samples of fondant candies to some extent, as follows:

- Potassium $(\mathrm{K})$ is found in the highest quantity in sample $1(65.08 \%)$ and the smallest quantity in sample $2(57.57 \%)$;

- Chlorine $(\mathrm{Cl})$ passes to the highest amount in sample $3(22.3 \%)$, and the smallest quantity is found in sample $1(15.2 \%)$;

- Phosphorus (P) is found in the highest quantity in sample $1(12.4 \%)$, and the smallest quantity in sample $2(10.84 \%)$;

- Calcium (Ca), sample 2 has the highest amount of calcium (8.52\%), and sample 3 - the smallest amount (3.06\%);

- Sulfur (S) migrates to the largest quantity in sample $2(2.22 \%)$, and the smallest quantity passes to sample $1(1.02 \%)$.

\subsection{Determination of reducing sugars. The Schoorl method}

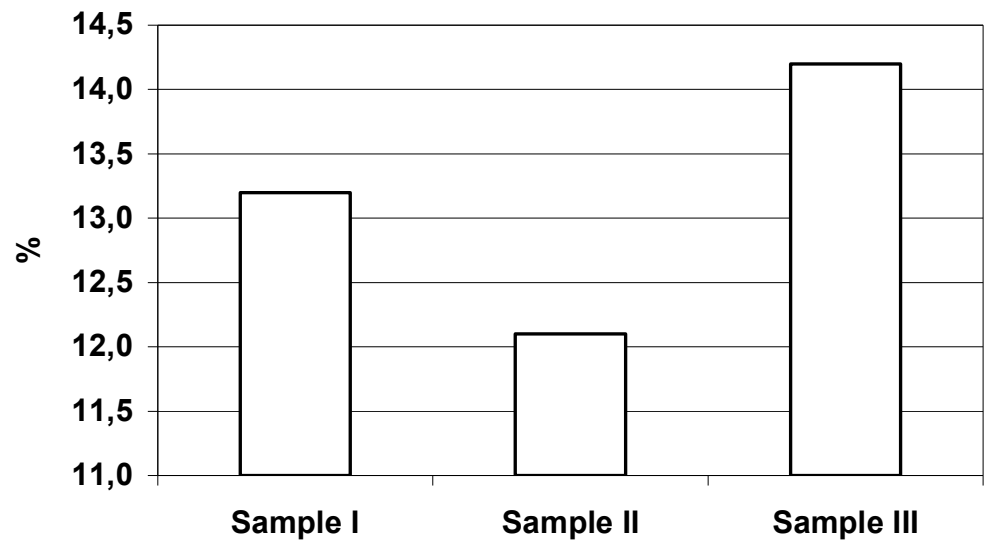

Figure 3.4. Graphical interpretation of reducing sugars content

Sample III has the highest amount of reducing sugars (14.2\%). Sample II has the lowest amount of reducing sugars (12.1\%). An amount of $13.2 \%$ was presented by sample I.

\section{Conclusions}

- $\quad$ Betaine (beetroot natural dye) can be used in both its liquid and powder form;

- Sample I (fondant candies with fresh beetroot juice) had the highest antioxidant activity and ash content;

- $\quad$ The highest moisture and amount of reducing sugars is given by sample III. Beetroot juice contains a high amount of mineral substances, but the heat treatment applied to the fondant candy affects the amount of minerals that migrate from the juice in the final product. 


\section{References}

1. Marques de Sá J.P. (2007), Applied Statistics using SPSS, STATISTICA, MATLAB and $R$, Second edition, Springer-Verlag Berlin Heidelberg.

2. James C. Griffiths (2013), Coloring Food \& Beverages.

3. Kanitha Ravichandran, Anja Kastell, Heidi Riedel, Iryna Smetanska (2011), Impact of processing of reed bett on betalain content and antioxidant acrivity, Food International.

4. Wild Flavors (2010), Natural Colors in Confectioneri.

5. Zhoeg Y.C.M., Corke H. (2015), Caracterization and aplication of betalain pigment from plants of Amaranthaceea, Trends in food Sience \& Technology.

6. Banu C., Janiţchi D., Jantea C., Bărăscu E. (2013), Tehnologia produselor zaharoase, Editura AGIR, Bucureşti.

7. Stone H., Bleibaum R., Thomas H.A. (2012), Sensory Evaluation Practices. Fourth Edition, Academic Press Elsevier.

8. Dildar A. Mehboob Khan M., Saeed R. (2015), Comparative Analysis of Phenolics, Flavonoids, and Antioxidant and Antibacterial Potential of Methanolic, Hexanic and Aqueous Extracts from Adiantum caudatum Leaves, Department of Chemistry, Forman Christian College, Pakistan.

9. Gatlan A. (2017), Aditivi alimentari. Determinarea activității antioxidante Metoda DPPH, Ștefan cel Mare University of Suceava, Romania, 2017

10. Showkat Ahmad Mir, S.M. Wani, Mukhtar Ahmad, Touseef Ahmed Wani, Adil Gani, S.A. Mir, F.A. Masoodi (2015), Effect of packaging and storage on the physicochemical and antioxidant properties of quince candy, India, 2015.

11. Buculei A. (2017), Determinarea conținutului de cenuşă din făină. Metoda calcinării, Ștefan cel Mare University of Suceava, Suceava.

12. Anton Slavov (2013), Antioxidant Activity of Red Beet Juices obtained after Micoweve and Termal Pretreatments.

13. Fiegal A. (2013), Scientists Make Reed Food Day from Potatoes, National Geographic. 